\title{
Expresión diferencial de la proteína LRBA en los linfocitos B vírgenes y de memoria humanos
}

\author{
Differential expression of LRBA protein in human naive and memory B lymphocytes
}

\begin{abstract}
Melissa Madrid ${ }^{1 *}$, Paula A. Restrepo ${ }^{1 *}$, Claudia M. Trujillo-Vargas ${ }^{1 *}$
Resumen

La deficiencia de LRBA (Lipopolysaccharide-responsive and beige-like anchor protein), es una inmunodeficiencia primaria causada por una expresión defectuosa de la proteína intracelular LRBA, debido a mutaciones, homocigotas y heterocigotas compuestas, en el gen correspondiente. Los pacientes con esta inmunodeficiencia muestran un amplio espectro de manifestaciones clínicas que incluyen, principalmente, inmunodeficiencia y autoinmunidad. La deficiencia de LRBA se asocia con una disminución del recuento de linfocitos B (LB) de memoria con cambio de isotipo en sangre periférica y plasmablastos, y una respuesta defectuosa de anticuerpos específicos, así como un defecto en la apoptosis y la autofagia. En este estudio se evaluó la expresión diferencial intracelular de la proteína LRBA en los LB vírgenes y de memoria de sangre periférica humana, bajo diferentes condiciones por citometría de flujo. Evidenciamos que los LB de memoria presentan una mayor expresión de LRBA; por otra parte, demostramos que la expresión de esta proteína en los LB aumenta considerablemente en condiciones de activación; sin embargo, la magnitud del incremento en la expresión de LRBA bajo esta condición es mayor en los LB vírgenes. En contraste, evidenciamos que la expresión de esta proteína disminuye en LB sometidos a apoptosis, aunque aún en estas condiciones, es mayor en LB de memoria. Estos hallazgos sugieren que LRBA juega un papel importante en el desarrollo de la memoria inmunológica humoral.
\end{abstract}

Palabras claves: apoptosis, citometría de flujo, inmunodeficiencias primarias, linfocitos B vírgenes, linfocitos B de memoria, ensayos de proliferación

\begin{abstract}
LRBA deficiency (Lipopolysaccharide-responsive and beige-like anchor protein) is a primary immunodeficiency caused by a defective expression of the intracellular protein LRBA, due to homozygous and heterozygous composed mutations in the corresponding gene. Patients with this immunodeficiency have a wide spectrum of clinical manifestations that include mainly immunodeficiency and autoimmunity. LRBA deficiency is associated with a decrease in class-switched memory B cells in peripheral blood and plasmablasts count, and a specific antibody response defective, as well as a defect in apoptosis and autophagy. In this study, intracellular differential expression of the LRBA protein in naïve and memory $B$ cells of human peripheral blood was evaluated under different conditions by flow cytometry. We evidence that memory $B$ cells have a higher expression of LRBA; on the other hand, we demonstrate that the expression of this protein in the $B$ cells increases considerably under activation conditions; however; the magnitude of the increase in LRBA expression under this condition is higher in naïve B cells. In contrast, we show that the expression of this protein decreases in $B$ cells undergoing to apoptosis, although even under these conditions, it is higher in memory $B$ cells. These findings suggest that LRBA plays an important role in the development of humoral immune memory.
\end{abstract}

Keywords: apoptosis, flow cytometry, primary immunodeficiency diseases, naïve B cells, memory B cells, proliferation assays

\footnotetext{
1. Grupo de Inmunodeficiencias primarias, Universidad de Antioquia. Medellín, Colombia.

* Autor de correspondencia: <melissa.madrid@udea.edu.co>; <paula.restrepo3@udea.edu.co>; <claudia.trujillo@udea.edu.co>
} 


\section{INTRODUCCIÓN}

Las inmunodeficiencias primarias son enfermedades raras y a menudo desconocidas, incluso para los profesionales de la salud y los médicos de atención primaria. Constituyen un grupo de aproximadamente 360 defectos genéticos del sistema inmunitario, tanto en sus componentes hematopoyéticos como en los no hematopoyéticos. Dichos defectos pueden presentarse con un amplio espectro de manifestaciones clínicas, incluyendo susceptibilidad incrementada a infecciones, inflamación, autoinmunidad o alergia. Hoy en día se estima que afectan a más de seis millones de personas alrededor del mundo, sin distinciones geográficas, de género o edad (Espinosa, Condino, Franco, y Sorensen, 2016).

La deficiencia de LRBA (Lipopolysaccharideresponsive and beige-like anchor protein), es una inmunodeficiencia primaria causada por mutaciones bialélicas en $L R B A$ que suprimen la expresión o afectan la función de esta proteína (Gámez-Díaz et al, 2016).

LRBA es una proteína de $300 \mathrm{kD}$, miembro de la familia de proteínas BDCPs $(B E A C H$ domain containing proteins) (Wang y Lockey, 2014), las cuales son altamente conservadas entre las especies y ampliamente expresadas en tejidos humanos inmunes y no inmunes (Cullinane, Schäffer, y Huizing, 2013).

En 2012, se describió por primera vez esta inmunodeficiencia en cinco pacientes pertenecientes a cuatro familias diferentes. Ese mismo año se identificaron otros pacientes con esta condición quienes presentaban, no solo inmunodeficiencia, sino también autoinmunidad (López et al, 2012).

Hoy en día se conoce que la deficiencia de LRBA es clínicamente variable. Entre las características fenotípicas autoinmunes que presentan estos individuos se incluyen: enteropatía autoinmune, enfermedad inflamatoria intestinal y diarrea no infecciosa, anemia hemolítica autoinmune y/o púrpura trombocitopénica inmune, entre otras. Por su parte, el fenotipo de inmunodeficiencia incluye hipogammaglobulinemia, organomegalia e infecciones recurrentes del tracto respiratorio principalmente (Alkhair et al, 2016), con una penetrancia variable (Gámez-Díaz et al, 2016).

Estudios de laboratorio, realizados en pacientes con deficiencia de LRBA, evidencian una disminución del recuento de Linfocitos B (LB) de memoria con cambio de isotipo y plasmablastos en sangre periférica, además de una respuesta defectuosa de anticuerpos específicos (Gámez-Díaz et al, 2016). Además, los LB de algunos de estos pacientes en cultivo muestran baja respuesta proliferativa, activación, diferenciación a plasmablastos y secreción de inmunoglobulinas (López et al, 2012).

Se ha demostrado que los individuos deficientes de $L R B A$ tienen defectos en la autofagia de los LB inducida por inanición y las líneas de LB inmortalizados de estos individuos muestran una susceptibilidad aumentada a la apoptosis (López et al, 2012; Revel et al, 2015); a su vez, se postula que los defectos observados en la producción de inmunoglobulinas, y la diferenciación y proliferación de los LB, podrían presentarse por el aumento de la muerte celular (López et al, 2012).

A pesar de todas estas evidencias, hasta la fecha no se ha comparado la expresión de LRBA en los LB vírgenes y de memoria humanos y tampoco se conoce si LRBA es importante para el desarrollo de la memoria inmunológica de LB. Por esta razón, el objetivo del presente estudio es evaluar in vitro la expresión de LRBA tanto en los LB vírgenes como de memoria de sangre periférica humana bajo diferentes condiciones.

\section{MATERIALES Y MÉTODOS}

Las células mononucleares de sangre periférica (CMSP) y LB utilizados en este estudio se aislaron, a partir de muestras de sangre periférica (SP) de nueve voluntarios sanos, en tubos heparinizados. Estos adultos no habían sido diagnosticados con inmunodeficiencias primarias y no presentaban infecciones, ni enfermedad aparente en el momento de la toma de muestra; la edad cronológica de los voluntarios oscilaba entre 23-32 años. Los donantes sanos firmaron el consentimiento informado aprobado según el protocolo 12-07-470 del Comité de Bioética de la Sede de Investigación Universitaria de la Universidad 
de Antioquia.

\section{Aislamiento de células mononucleares de sangre periférica}

Las CMSP se aislaron a partir de muestras de SP de voluntarios adultos sanos en tubos heparinizados y se obtuvieron mediante gradiente de densidad de Ficoll (Histopaque-1077 ${ }^{\mathrm{TM}}$ Sigma-Aldrich, St. Louis, MO). En breve, la sangre se centrifugó a 1800 r. p. m. por $5 \mathrm{~min}$, para luego tomar la interfase en la cual se encontraba el buffy coat o capa de leucocitos; esta se mezcló con $4 \mathrm{~mL}$ de PBS Dulbecco (Sigma-Aldrich), para luego adicionarla suavemente a $3 \mathrm{~mL}$ de Ficoll y centrifugar a $1200 \mathrm{~g}$ por $20 \mathrm{~min}$. Finalmente, se separó la capa de CMSP de la interfase entre el PBS y el ficoll, después de dos lavados se realizó el conteo y se evaluó la viabilidad celular mediante exclusión con azul de tripano (Sigma-Aldrich).

\section{Enriquecimiento de linfocitos B humanos totales y separación celular electromagnética en linfoci- tos $\mathrm{B}$ vírgenes y de memoria}

Se recolectó sangre de voluntarios adultos sanos en tubos heparinizados y se enriquecieron los LB mediante el coctel de enriquecimiento de LB RosetteSep $^{\mathrm{TM}}$ (Stemcell Technologies, Vancouver, Canadá) por selección negativa, siguiendo las instrucciones del fabricante. Se realizó el conteo de la suspensión celular obtenida y se evaluó la viabilidad celular mediante exclusión con azul de tripano.

Los LB humanos obtenidos por el enriquecimiento fueron marcados fluorescentemente con los anticuerpos monoclonales anti-CD19 (Clon: SJ25C1, Becton Dickinson, BD, Franklin Lakes, NJ) y antiCD27 (Clon: 0323, eBioscience, Waltham, MA), por 30 min protegidos de la luz. Luego, las células se resuspendieron en $2 \mathrm{~mL}$ de medio RPMI (SigmaAldrich, San Luis, Misuri) suplementado con suero fetal bovino (SFB), al $10 \%$ penicilina y estreptomicina al 2\% (Gibco life technologies) (Medio RPMI completo), para posteriormente separar las subpoblaciones de LB vírgenes (CD19+/CD27-) y de memoria (CD19+/CD27+) mediante separación celular electromagnética usando un MoFlo XDP (Beckman Coulter, Indianapolis) bajo condiciones estériles.
La pureza del enriquecimiento y de la separación celular electromagnética se determinó por citometría de flujo inmediatamente después de realizar cada proceso.

\section{Activación celular}

Tanto las CMSP como los LB vírgenes y de memoria altamente purificados se cultivaron en presencia o ausencia de $0,3 \mu \mathrm{L}$ de un trímero de CD40 ligando (tri-CD40L) e interleuquina 21 (IL-21) recombinantes (reactivos utilizados directamente a partir de los sobrenadantes obtenidos de los cultivos de células transfectadas con los respectivos plásmidos, generosamente donados por Hermann Eibel, University Medical Center Freiburg, Center for Chronic Immunodeficiency (CCI), Friburgo, Alemania), a una densidad celular final de 1x105 células en un volumen de $200 \mu \mathrm{L} /$ pozo. Estas células fueron suspendidas en medio RPMI completo, en platos de 96 pozos fondo $\mathrm{U}$, durante cinco días a $37{ }^{\circ} \mathrm{C}$ bajo atmósfera al $5 \%$ de $\mathrm{CO}_{2}$. Luego, se procedió a evaluar la expresión de la proteína LRBA por citometría de flujo, como se describirá posteriormente.

Para confirmar la eficiencia de la estimulación con el trí-CD40L+IL-21, se evaluó la proliferación celular por citometría de flujo. Para esto se cultivaron las células sin y con estímulo, previamente marcadas con éster de succinimidil-carboxifluoresceína (CFSE, $5 \mu \mathrm{M}$, Molecular Probes, Eugene, OR) por cinco días. Para excluir las células muertas después de la incubación, se realizó una tinción con $100 \mu \mathrm{L}$ de una dilución de Live/Dead Fixable Aqua Dead Cell Stair (Aqua $^{\circledR}$, Molecular probes ${ }^{\mathrm{TM}}$ ) 1:1000 en PBS.

\section{Inducción de apoptosis}

La apoptosis se indujo mediante el uso de estaurosporina (Sigma-Aldrich) $(4 \mu \mathrm{M})$, preparada en medio RPMI sin suplementar, a una densidad celular final de 1 x105 células en un volumen de $200 \mu \mathrm{L} /$ pozo durante 6 h. Como control negativo, se usaron células no expuestas al inductor. Inmediatamente después del tratamiento con estaurosporina se procedió a evaluar la presencia de apoptosis y la expresión de la proteína LRBA.

Para evaluar la apoptosis, en ensayos preliminares 
se realizó la tinción ioduro de propidio/Anexina $\mathrm{V}$ marcada fluorescentemente (BD); sin embargo; por cuestiones de solapamiento entre algunos fluorocromos, se decidió sustituir el ioduro de propidio por Aqua $^{\circledR}$ con el fin de excluir las células muertas al momento del análisis. Así que en la mayoría de los ensayos las células se lavaron con PBS y se tiñeron con $100 \mu \mathrm{L}$ de una dilución de Aqua ${ }^{\circledR}$ 1:1000 en PBS (por 15 min, a $4{ }^{\circ} \mathrm{C}$, protegido de la luz). Posteriormente, se realizó un lavado con PBS y dos lavados con $100 \mu \mathrm{L}$ de tampón de unión (Binding buffer) 1X (BD) y se determinó la apoptosis mediante tinción con $5 \mu \mathrm{L}$ Annexina $\mathrm{V}$, por 15 min a temperatura ambiente. Finalmente, se realizó un lavado con 400 $\mu \mathrm{L}$ de Binding buffer 1X. La intensidad de las células Anexina $\mathrm{V}$ positivas se determinó en un citómetro de flujo LSR Fortessa (BD).

\section{Análisis de la expresión de LRBA}

Para evaluar la expresión de la proteína LRBA, las células se lavaron con PBS a 1800 r. p. m. por 5 min y se tiñeron con los anticuerpos anti-CD45 (clon: 2D1, e-Bioscience, Waltham, MA) anti-CD19 y anti-CD27 marcados fluorescentemente por 30 min protegidos de la luz a $4{ }^{\circ} \mathrm{C}$. En el caso de los LB, obtenidos mediante enriquecimiento y separación celular electromagnética, no fue necesario volver a realizar esta tinción, ya que las células habían sido marcadas previamente.

Luego, las células se fijaron y permeabilizaron con $200 \mu \mathrm{L}$ de la solución Cytofix/Cytoperm ${ }^{\mathrm{TM}}$ (BD) por $20 \mathrm{~min}$ a $4{ }^{\circ} \mathrm{C}$. Después de dos lavados con $200 \mu \mathrm{L}$ del tampón Perm/Wash ${ }^{\mathrm{TM}}$ (1X) (BD), a las células se les adicionaron $50 \mu \mathrm{L}$ de una dilución 1:160 del anticuerpo primario anti-LRBA (HPA 019366, Sigma-Aldrich) y se incubó por $30 \mathrm{~min}$, protegido de la luz. Se lavaron nuevamente con el tampón Perm/Wash 1X y finalmente se realizó la tinción con $50 \mu \mathrm{L}$ de una dilución 1:3200 del anticuerpo secundario goat anti-rabbit IgG anti-LRBA marcado fluorescentemente (Molecular Probes ${ }^{\mathrm{TM}}$ ) por $30 \mathrm{~min}$, protegido de la luz. La dilución de los anticuerpos se realizó en Perm/Wash 1X.

Las diluciones de los anticuerpos fueron escogidas después de realizar una titulación de ambos, ya que en las diluciones recomendadas (anticuerpo primario 1:80 y anticuerpo secundario 1:800) se observaron uniones inespecíficas.

Finalmente, la expresión de la proteína LRBA se evaluó también en el citómetro LSRFortessa ${ }^{\mathrm{TM}}$.

\section{RESULTADOS}

\section{La separación de las subpoblaciones de linfocitos B logra un óptimo enriquecimiento celular}

Para esto se emplearon diferentes metodologías que, a mayor complejidad, se esperaba lograran un mayor enriquecimiento celular, aspecto que siempre se verificó por citometría de flujo usando anticuerpos marcados fluorescentemente, dirigidos contra moléculas transmembranales, característicamente expresadas por las subpoblaciones de LB objeto de este estudio como CD45, CD19 y CD27 (Antunes, Kaski, y Dumitriu, 2012; Klein, Rajewsky, y Küppers, 1998). Nosotros separamos, en primer lugar, las CMSP (linfocitos y monocitos) por medio de un gradiente de densidad sobre Ficoll. En la figura 1A se muestran los datos representativos de los experimentos en los cuales se discriminaron los LB vírgenes y de memoria a partir de CMSP, presentando a su vez la estrategia de gating utilizada. En primer lugar, se discriminaron los leucocitos, de acuerdo con su tamaño (Forward Scatter, FSC) y granularidad (Side Scatter, SSC), de los eritrocitos, células muertas y detritos que pueden quedar después de realizar la separación. Luego de la exclusión de agregados, usando los parámetros FSC-A (Forward Scatter Area) y FSC-H (Forward Scatter Height), se seleccionaron los leucocitos basados en la expresión de CD45, para luego separar, en los análisis, los LB (CD19+). Seguidamente, basándonos en la expresión de CD27, se discriminaron los LB vírgenes (CD19+/CD27-) y de memoria (CD19+/CD27+). En los ensayos realizados con donantes sanos, los LB correspondieron aproximadamente al 5,9\% (4,5-7,1\%) del total de los leucocitos (todos los datos presentados en esta sección se refieren a la Mediana (Min-Max)), y los LB vírgenes y de memoria correspondieron al 73,9\% $(67,0-80,0 \%)$ y $26,1 \%(19,8-33,0 \%)$ del total de los LB, respectivamente. Esto concuerda con los valores de referencia en SP humana reportados por Morbach, 
Eichhorn, Liese, y Girschick (2010).

En la figura 1B se observa un ejemplo de los resultados obtenidos usando el cóctel de enriquecimiento, basado en la selección negativa (RosetteSep o rosetas) de LB (CD19+) en SP. En los doce experimentos realizados mediante esta técnica, se observó que el porcentaje de LB en SP humana era aproximadamente de $6,2 \%(3,8-7,8 \%)$ del total de leucocitos antes de emplear el cóctel; este valor pasó a ser aproximadamente 87,8 \% (81,4-97,3\%) del total de leucocitos luego de utilizar RosetteSep.

Después de obtener los LB totales CD19+, en nueve experimentos se separaron en las subpoblaciones CD27+ y CD27- mediante separación electromagnética (figura 1C). Los resultados de estas nueve separaciones electromagnéticas indicaron que el porcentaje de LB vírgenes del total de LB en SP corresponde a 70,6 (60,0-81,7\%) y que el porcentaje de LB de memoria fue de 29,7\% (18,2-40,0\%), valores que se asemejan a lo obtenido por la técnica de Ficoll. Además, utilizando este método de separación, obtuvimos rangos de pureza para los LB vírgenes de $96,8 \%(94,0-97,9 \%)$ y para los LB de memoria de $92,8 \%(90,9-93,8 \%)$.

Otro dato interesante de estos experimentos es el rendimiento celular, es decir, la cantidad de células de la población de interés que es posible obtener por $\mathrm{mL}$ de sangre. Utilizando el gradiente de densidad de ficoll, el rendimiento en nuestros experimentos fue de $1,5 \times 10^{6} \operatorname{CMSP}\left(1,4-2,0 \times 10^{6}\right)$, al utilizar las rosetas fue de 195.312 LB (103.125-315.000) y al utilizar la separación electromagnética fue de 92.400 para los LB vírgenes (34.742-460.000) y para los LB de memoria fue de 46.900 (14.928-67.524).

\section{Estimulación e inducción de apoptosis en los lin- focitos B}

Después de asegurar las condiciones óptimas para obtener una adecuada separación, pureza y rendimiento celular, y de establecer las estrategias de gating, nuestros siguientes experimentos estaban encaminados en lograr la activación celular de los LB. Para esto decidimos emplear como estímulo tri-CD40L e IL-21. $\mathrm{Al}$ usar un trímero de CD40L in vitro se logra la activación de las LB ya que se facilita el entrecruzamien- to de estos receptores, aumentando así la población celular (Korniluk, Kemona, y Dymicka-Piekarska, 2014). La IL-21 por su parte, interacciona con un receptor heterodímerico (IL21R), desempeñando un papel clave en la proliferación y diferenciación de los LB previamente estimulados por linfocitos $\mathrm{T}$, facilitando la formación de los centros germinales y la producción de anticuerpos (Good, Bryant, y Tangye, 2006). Según estudios previos, el sinergismo entre CD40L + IL-21 in vitro es potente para inducir una proliferación extensa de los LB (Marasco et al, 2017).

En la figura 2 presentamos un ejemplo representativo de la proliferación de LB totales en CMSP luego del estímulo empleado. Después de la selección de las células vivas (AQUAneg), se seleccionaron los LB $(\mathrm{CD} 19+)$ y se evaluó en ellos el efecto de dilución del marcador CFSE debido a la proliferación. Observamos un menor número de células vivas después del estímulo, posiblemente debido al fenómeno de muerte inducida por activación que se ha descrito en otros estudios (Brenner et al, 2007). Las células activadas también aumentaron su granularidad (observada por el parámetro SSC-A) y en efecto, distribuyeron el marcador CFSE a las células hijas (figura 2B).

En la tabla 1 se presenta un resumen de los resultados de todos los experimentos de proliferación. Estos fueron realizados tanto en CMSP como en LB separados por rosetas y en LB vírgenes obtenidos luego de la separación electromagnética. No fue posible evaluar la proliferación en LB de memoria purificados debido a que no se obtuvo la cantidad suficiente para este ensayo. Es interesante observar que en los LB totales obtenidos a partir de CMSP, la proliferación es mayor que en aquellos LB totales aislados por rosetas, lo cual sugiere la importancia de células accesorias en el cultivo para lograr una mayor proliferación de LB. También, con las condiciones utilizadas en este estudio para inducir la proliferación, se logró un mayor porcentaje de células en división en los LB de memoria, que en los LB vírgenes en CMSP.

En estudios previos se registra que la proteína LRBA juega un papel en la regulación de la apoptosis (Revel et al, 2015). Por lo tanto, nuestros siguientes experimentos estuvieron enfocados en inducir apoptosis en los LB, para así posteriormente poder evaluar la 
A.

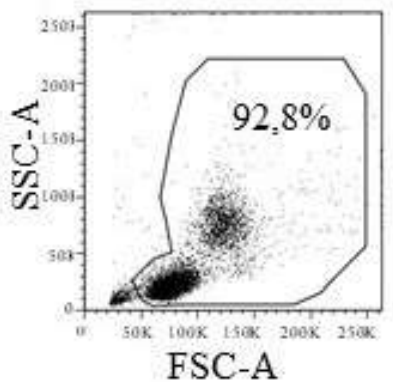

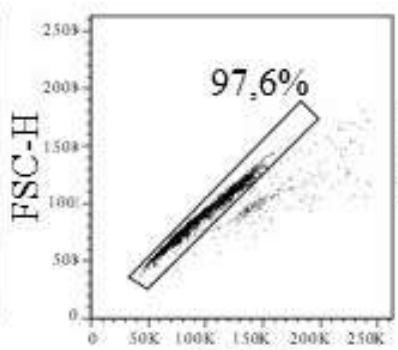

FSC-A

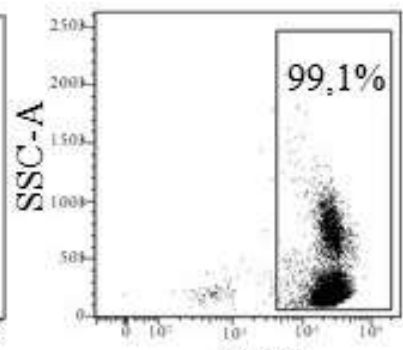

CD45
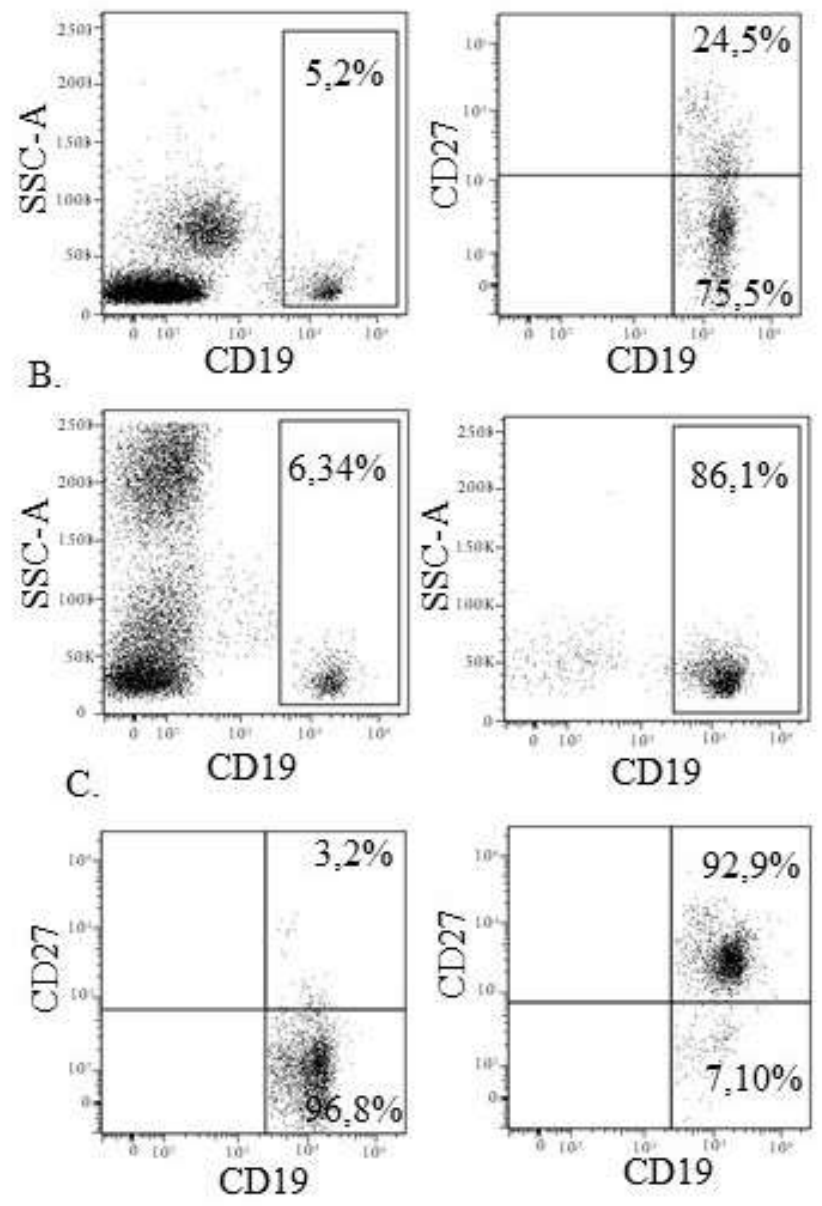

Figura 1. Separación de linfocitos B por diferentes metodologías. A. Ejemplo representativo de la distribución de las células mononucleares de sangre periférica de acuerdo con su tamaño (FSC-A) y granularidad (SSC-A). Luego de la exclusión de agregados mediante el uso de los parámetros FSC-A y FSC-H, los linfocitos B se separaron para su análisis por medio de la expresión de CD45 y CD19. Finalmente se analizó la expresión de CD27 para discriminar los linfocitos B vírgenes (CD19+/CD27-) y de memoria (CD19+/CD27+). B. Ejemplo representativo de los linfocitos $B$ de sangre periférica obtenidos antes (izquierda) y después (derecha) del enriquecimiento con las rosetas o $\mathrm{C}$. Por separación electromagnética, para obtener las subpoblaciones de linfocitos B vírgenes (CD27 negativo, izquierda) y de memoria (CD27 positivo, derecha). 

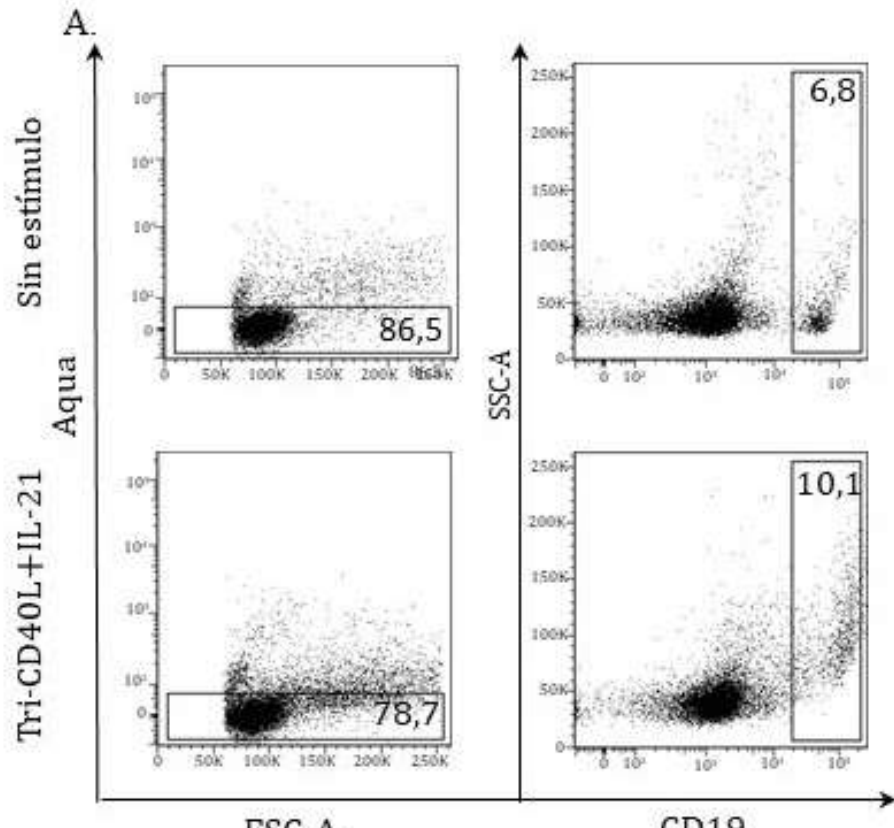

B.

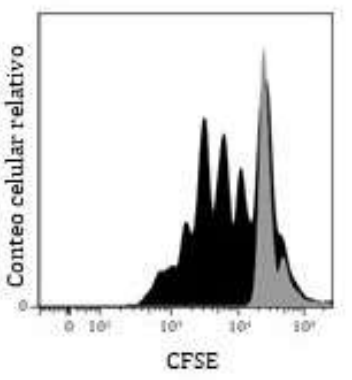

C.

FSC-A

CD19

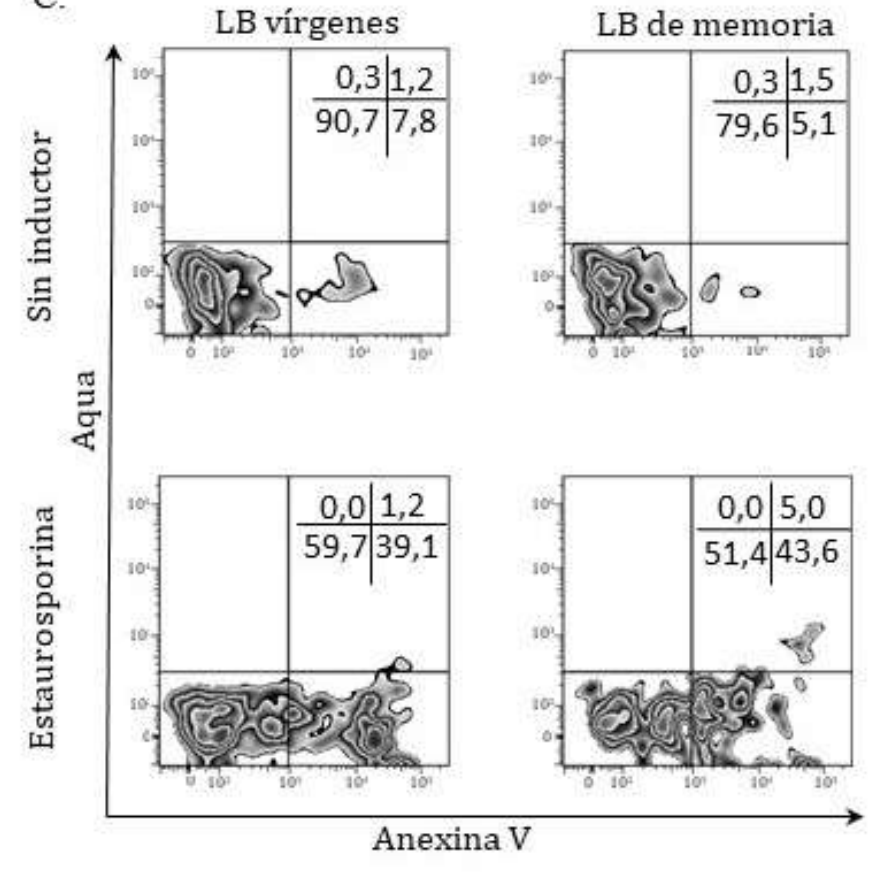

Figura 2. Evaluación de la proliferación y apoptosis en los linfocitos B humanos. Ejemplo representativo en linfocitos B totales. A. Luego de la exclusión de las células muertas (AQUA ${ }^{\circledR}$ positivas) se separaron los linfocitos B para su análisis por medio de la expresión de CD19. B. Se evaluó la proliferación de las células mediante el ensayo de dilución de fluorescencia del CFSE. En gris se presentan las células sin estímulo y en negro aquellas estimuladas. $C$. Ejemplo representativo de la apoptosis en linfocitos $B$ vírgenes y de memoria provenientes de CMSP, en condiciones basales y expuestos a la estaurosporina. 
expresión de la proteína LRBA tanto en LB vírgenes como de memoria, bajo condiciones de apoptosis. La estaurosporina, producto natural aislado de la bacteria Streptomyces staurosporeus, es un inhibidor no selectivo de la proteína quinasa $\mathrm{C}$ que induce apoptosis mediante la vía intrínseca o mitocondrial (Antonsson y Persson, 2009).

En la figura $1 \mathrm{C}$ se presenta un ejemplo representativo de los ensayos de apoptosis en los LB vírgenes y de memoria; en tabla 2 se presenta un resumen de los resultados de todos los experimentos de apoptosis realizados en nuestro estudio, permitiéndonos así comparar el porcentaje de apoptosis neta lograda en $\mathrm{LB}$ vírgenes y de memoria obtenidas a partir de CMSP y por separación electromagnética. Es interesante observar que los LB de memoria son más susceptibles a la apoptosis inducida por la estau- rosporina que los vírgenes. Además, se observó que al parecer la separación electromagnética afecta la viabilidad celular ya que se obtiene un porcentaje mayor tanto de células apoptóticas como necróticas en comparación a cuando las células se obtienen a partir de CMSP. Con respecto a la necrosis, estos resultados confirman el efecto deletéreo de la separación electromagnética sobre la viabilidad celular de los LB vírgenes y de memoria tanto en condiciones basales como luego de la exposición a estaurosporina. En el caso de la susceptibilidad celular a la apoptosis, aumentada después de la separación electromagnética en estas subpoblaciones de LB, podría deberse a un efecto más directo de la estaurosporina sobre estas células en ausencia de otras células accesorias presentes en las CMSP o a la ausencia de factores de sobrevida que estas células accesorias proporcionan en la suspensión celular.

Tabla 1. Resumen de los ensayos de proliferación en linfocitos B totales, vírgenes y de memoria obtenidos a partir de CMSP, rosetas y separación electromagnética.

\begin{tabular}{l|l|l|l}
\hline \multicolumn{2}{l|}{ Técnica de obtención de linfocitos } & $\boldsymbol{n}$ & Porcentaje de células divididas * \\
\hline \multirow{3}{*}{ CMSP } & LB & 4 & $33,4(15,7-55,1)$ \\
& LB CD27- & 2 & $7,4(2,5-12,4)$ \\
& LB CD27+ & 2 & $24,9(21,9-27,9)$ \\
\hline Rosetas & LB & 6 & $15,8(6,7-18,3)$ \\
\hline Separación electromagnética & LB CD27** & 2 & $10,5(10,4-10,6)$ \\
\hline
\end{tabular}

LB: Linfocitos B. $n=$ número de experimentos realizados y tabulados.

* En el porcentaje de células en división se presenta la Mediana (Min-Max) después de sustraer el porcentaje de células divididas en condiciones basales.

** Los LB CD27- se obtuvieron por separación electromagnética de LB enriquecidos por la técnica de rosetas.

Tabla 2. Resumen de los ensayos de apoptosis en linfocitos B vírgenes y de memoria obtenidos a partir de CMSP y separación electromagnética.

\begin{tabular}{l|l|l|l|l}
\hline \multicolumn{2}{l|}{ Técnica de obtención de linfocitos } & $\boldsymbol{n}$ & $\%$ apoptóticas * & $\%$ necróticas ** \\
\hline \multirow{2}{*}{ CMSP } & LB CD27- & 4 & $19,3(10,9-31,3)$ & Indet. \\
& LB CD27+ & 4 & $23,7(20,7-38,5)$ & Indet. \\
\hline \multirow{2}{*}{ Separación electromagnética } & LB CD27- & 3 & $30,5(12,2-30,7)$ & $9,8(3,1-10,3)$ \\
& LB CD27+ & 3 & $36,1(31,2-47,2)$ & $10,0(9,8-17,8)$ \\
\hline
\end{tabular}

LB: Linfocitos B. $n=$ número de experimentos realizados y tabulados. Indet. = Indetectable

* En el porcentaje de células apoptóticas se presenta la Mediana (Min-Max) después de sustraer el porcentaje de células apoptóticas en condiciones basales.

** En el porcentaje de células necróticas se presenta la Mediana (Min-Max) después de sustraer el porcentaje de células necróticas en condiciones basales.

Células apoptóticas: Células anexina $\mathrm{V}+\mathrm{y}$ Aqua ${ }^{\circledR}$ negativas.

Células necróticas: Células anexina $\mathrm{V}+$ Aqua $^{\circledR}+$ 


\section{La proteína LRBA es diferencialmente expresada en linfocitos $B$ vírgenes y de memoria}

López et al (2012) demostraron que los pacientes con deficiencia de LRBA muestran alteraciones en la inmunidad humoral y tienen un número alterado de las subpoblaciones de LB en SP. Por lo tanto, inicialmente se evaluó si la expresión de LRBA se modificaba en LB activados (expuestos al tri-CD40L e IL-21) de adultos sanos. En la figura 3A se muestra un ejemplo representativo de los experimentos realizados, en los cuales se observa que los LB de SP humana de los controles expresan intracelularmente LRBA sin estar expuestos a ningún estímulo. Sin embargo, la intensidad media de fluorescencia (IMF) de LRBA aproximadamente se duplica en LB expuestos al estímulo. En la figura 3B se grafican las IMF de los tres experimentos realizados, demostrando la reproducibilidad de este hallazgo.

Posteriormente, decidimos separar los LB vírgenes y de memoria a partir de CMSP para luego activar los LB como se describió anteriormente y evaluar la expresión de LRBA en ambas subpoblaciones. En la figura $4 \mathrm{~A}$ se muestra un ejemplo representativo de los experimentos realizados, en los cuales observamos que la IMF de LRBA aumenta tanto en los LB vírgenes y de memoria cuando estos se exponen al tríCD40L e IL-21. En la figura 4B están representados las IMF de los tres experimentos realizados en los cuales comprobamos que en todos nuestros experimentos hubo un incremento en la expresión de LRBA en ambas subpoblaciones cuando son expuestas al estímulo utilizado.

Aunque en los experimentos anteriores observamos la regulación positiva en la expresión de LRBA en LB activados, no se observó una diferencia significativa en la expresión de este marcador entre LB vírgenes y de memoria. Esto debido principalmente a la fluctuación en los valores de IMF en los diferentes experimentos y al solapamiento de los datos. Sin embargo, analizando cada experimento por separado, siempre se observó una mayor expresión de LRBA (mayor IMF) en LB de memoria. Para presentar este hallazgo, calculamos la razón de la IMF de la expresión de LRBA en los LB de memoria/LB vírgenes; este índice permite determinar qué tanto más se expresa
LRBA en LB de memoria con respecto a los vírgenes: valores iguales a 1 indican que la expresión en ambas subpoblaciones es igual, mayores de 1 indican que la expresión es mayor en LB de memoria y menores de 1 que la expresión es mayor en los LB vírgenes. En la figura 4C están graficados los datos obtenidos de los experimentos realizados en CMSP. Se observó que cuando las CMSP no son expuestas al estímulo sino únicamente incubadas por cinco días en medio RPMI completo, los LB de memoria siempre presentaron una mayor expresión de LRBA en comparación con los LB vírgenes. Sin embargo, tras el estímulo, aunque la expresión de LRBA sigue siendo mayor en los LB de memoria, esta razón se acerca más a 1. De manera interesante, cuando esto mismo se evalúa en LB obtenidos mediante separación celular electromagnética, estas diferencias no se conservan, aunque la mayoría de las veces se observó una mayor expresión de LRBA en LB de memoria.

Para confirmar estos hallazgos, decidimos entonces evaluar la expresión de LRBA en LB en condiciones basales (ex vivo) ya sea en CMSP o después de la separación electromagnética. En la figura 4D se observa que en condiciones basales en CMSP, los LB de memoria siempre tienen una mayor expresión de LRBA que los vírgenes y de nuevo, esta diferencia no se conserva cuando los LB son separados electromagnéticamente.

Después de conocer que el estímulo tri-CD40L e IL-21 aumentó la expresión de la proteína LRBA tanto en los LB vírgenes y de memoria, decidimos evaluar en cuál de las dos subpoblaciones de LB el estímulo utilizado tenía un mayor efecto en modular positivamente esta proteína intracelular. Para esto, calculamos la razón de la IMF de la expresión de LR$\mathrm{BA}$ en los LB con estimulo/IMF en LB sin estímulo. Así determinamos el incremento en la expresión de LRBA en las células con estímulo comparadas con las no estimuladas en ambas subpoblaciones de LB. De la misma manera que explicamos anteriormente, valores iguales a 1 indican que la expresión es igual tanto en las células expuestas al estímulo como sin este; valores mayores a 1 indican que la expresión es mayor en células expuestas al estímulo y menores a 1 indican que es mayor en células sin estímulo. Aunque ya habíamos mostrado anteriormente que la 
A.

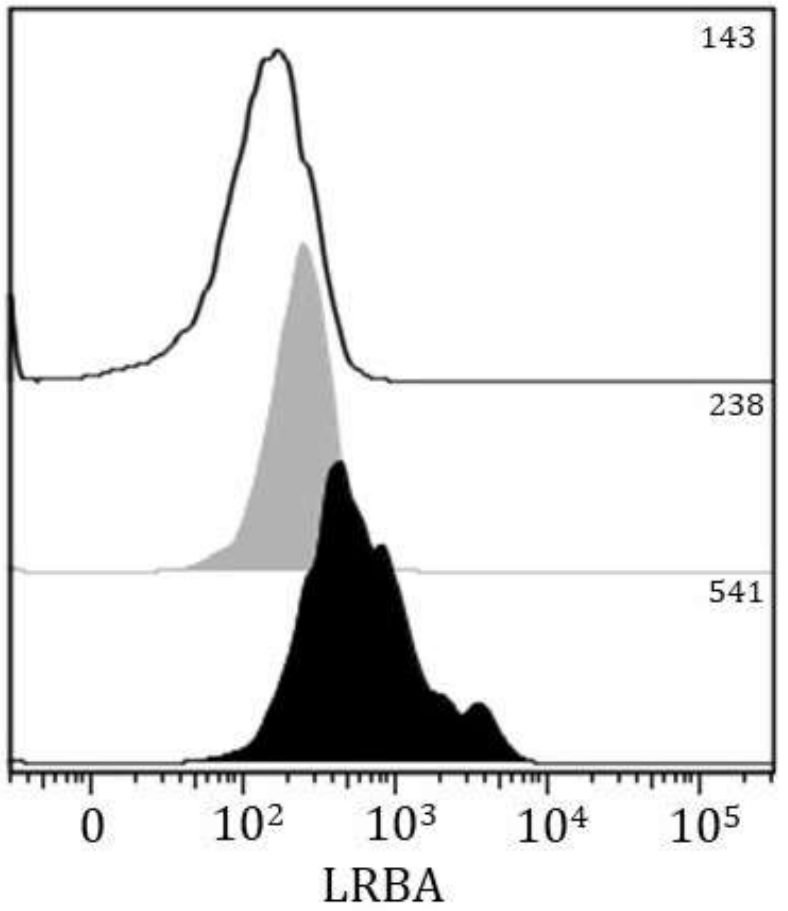

B.

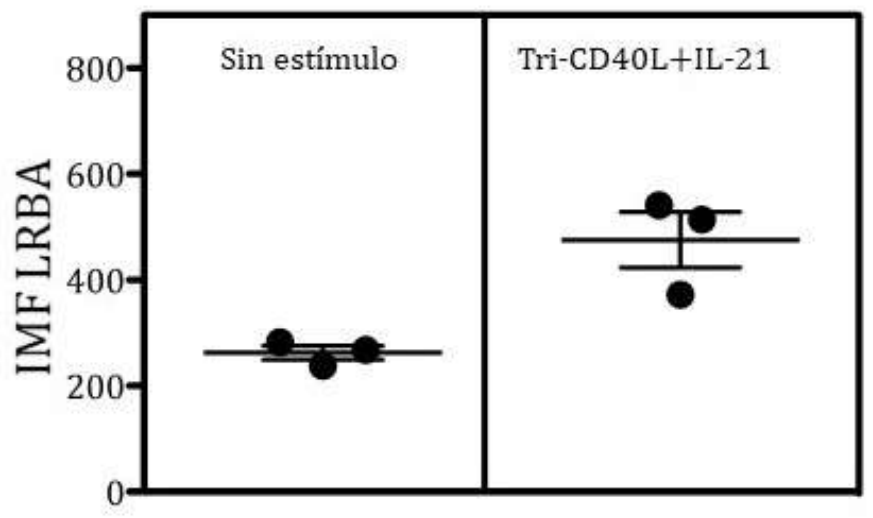

Figura 3. Expresión de LRBA en linfocitos $B$ totales de sangre periférica estimulados con el trímero CD40L e IL-21 recombinantes. A. Ejemplo representativo de la expresión de LRBA en linfocitos B totales de sangre periférica obtenidos mediante el cóctel de enriquecimiento e incubados por cinco días sin o con el trímero CD40L e IL-21. El histograma solo delineado corresponde al resultado de la tinción únicamente con el anticuerpo secundario (control negativo), el gris a los linfocitos B no expuestos al estímulo y el negro a aquellos estimulados. Los números en la parte superior derecha de cada gráfica corresponden a la intensidad media de fluorescencia (IMF) de LRBA. B. IMF de LRBA en los tres experimentos realizados. 
A.

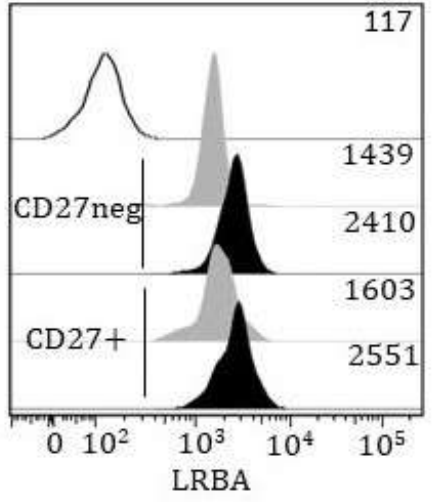

C.

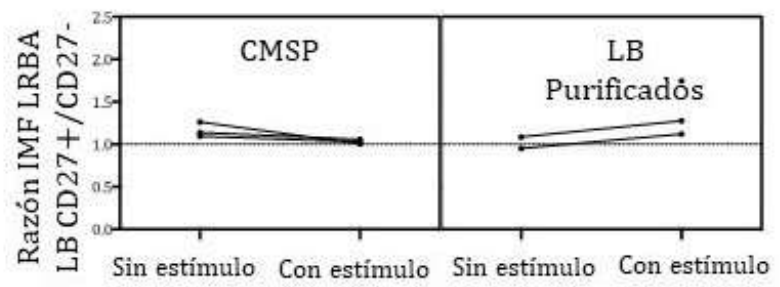

B.

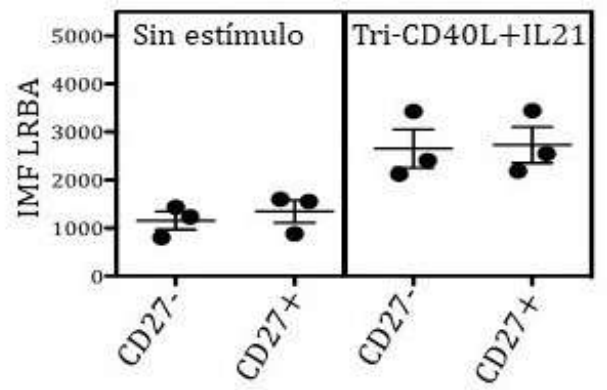

D.

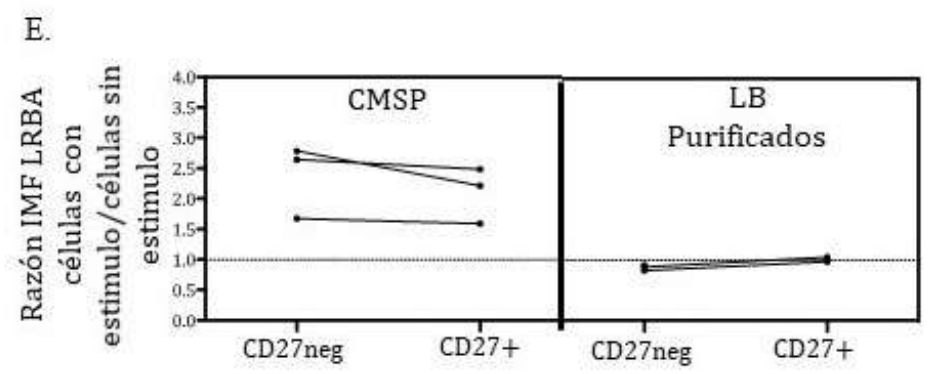

Figura 4. Expresión de LRBA linfocitos B vírgenes y de memoria obtenidas a partir de células mononucleares de sangre periférica, estimulados con el trímero CD40L e IL-21. A. Ejemplo representativo de la expresión de LRBA en linfocitos B vírgenes (CD27neg) y de memoria (CD27+) obtenidos a partir de células mononucleares de sangre periférica incubadas por 5 días en ausencia o presencia del trímero CD40L e IL-21. El histograma solo delineado corresponde a la tinción únicamente con el anticuerpo secundario, los histogramas grises representan los resultados en linfocitos B no expuestos al estímulo y los histogramas negros en aquellos activados. B. Intensidad media de fluorescencia de los resultados obtenidos en los tres experimentos realizados. C. Razón de la intensidad media de fluorescencia de LRBA en linfocitos B de memoria/linfocitos B vírgenes en ausencia (sin estímulo) o presencia (con estímulo) del trímero CD40L e IL21 por cinco días, ya sea en linfocitos $B$ a partir de células mononucleares de sangre periférica o purificados por separación electromagnética. D. Razón de la intensidad media de fluorescencia de LRBA en linfocitos B de memoria/linfocitos B vírgenes en condiciones basales (ex vivo), ya sea en linfocitos B a partir de células mononucleares de sangre periférica o purificados por separación electromagnética. $E$. Razón de la intensidad media de fluorescencia de LRBA en linfocitos B vírgenes (CD27-) y de memoria (CD27+) expuestos al estímulo/aquellos sin el estímulo por cinco días, ya sea en linfocitos B a partir de células mononucleares de sangre periférica o purificados por separación electromagnética. Los resultados de cada experimento se encuentran unidos por una línea. La línea punteada indica igual expresión de LRBA en ambas subpoblaciones de LB. 
expresión de LRBA es mayor en LB de memoria provenientes de CMSP, en la figura $4 \mathrm{E}$ se observa que el tri-CD40L e IL-21 tiene un mayor impacto en el aumento de la expresión de LRBA en los LB vírgenes (CD27 neg) provenientes de CMSP. Sin embargo, cuando los LB son purificados mediante separación electromagnética, estas diferencias no se conservan.

\section{La inducción de apoptosis en Linfocitos B dismi- nuye la expresión de LRBA, aunque se sigue ob- servando una mayor expresión de esta proteína en LB de memoria}

En la figura 5A se muestra un ejemplo representativo de los tres experimentos realizados induciendo apoptosis en los LB, en los cuales observamos que la IMF de la proteína LRBA disminuye tanto en los LB vírgenes como de memoria cuando estas células se exponen a estaurosporina. En la figura $5 \mathrm{~B}$ se grafican las IMF de todos los experimentos realizados que confirman que hubo una disminución en la expresión de LRBA en ambas subpoblaciones de LB bajo las condiciones de apoptosis establecidas. Para confirmar que este hallazgo se conserva en todos los experimentos realizados, en la figura $5 \mathrm{C}$ graficamos nuevamente los datos de la razón de la IMF de los LB expuestos a estaurosporina/IMF de los LB no expuestos. En esta figura es posible observar que en $\mathrm{LB}$ vírgenes y de memoria obtenidos tanto a partir de CMSP como por separación celular electromagnética, la expresión de LRBA siempre se modula negativamente (Razón $<1$ ) cuando las células son expuestas a estaurosporina. Luego evaluamos si había una diferencia en la expresión de LRBA entre LB vírgenes y de memoria bajo condiciones de apoptosis; para esto calculamos la razón de la IMF de la expresión de LRBA en los LB de memoria/LB vírgenes analizando cada experimento por separado. En la figura de la izquierda de la figura 5D se presentan los datos obtenidos de los experimentos realizados en CMSP. Se observa que los LB de memoria siempre presentaron una mayor expresión de la proteína LRBA en comparación con los LB vírgenes tanto en las células no expuestas al inductor como en aquellas expuestas a estaurosporina; sin embargo, cuando los LB son obtenidos mediante separación celular electromagnética estas diferencias no se observan. Entonces, confirmamos que hay una mayor expresión de LRBA en LB de memoria tanto en ausencia como en presencia de la estaurosporina, en presencia de células accesorias (es decir, en CMSP totales), más no en células purificadas. Además, la expresión de LRBA disminuye tras la exposición a estaurosporina.

\section{DISCUSIÓN}

El objetivo del presente estudio fue evaluar la expresión de LRBA tanto en los LB vírgenes como de memoria de sangre periférica humana bajo diferentes condiciones. Inicialmente confirmamos que la expresión de LRBA aumenta en LB estimulados con un trímero de CD40L+IL-21 y disminuye en aquellos sometidos a apoptosis inducida por estaurosporina, tal como se había reportado previamente (GámezDíaz et al, 2018). En general, la expresión de esta proteína intracelular es mayor en los LB de memoria que en los vírgenes (tanto ex vivo, como sometidos a estimulación o apoptosis). Sin embargo, la intensidad de esta diferencia depende de si estas células se analizan directamente de CMSP o si son separadas electromagnéticamente. También observamos que el impacto del cambio en la expresión de LRBA en las células estimuladas es mayor en LB vírgenes. Estos resultados apoyan estudios previos que sugieren la importancia de LRBA para el desarrollo de la memoria inmunológica humoral (Revel et al, 2015).

Para separar LB vírgenes y de memoria nosotros usamos el CD27, que en el caso de los LB es inducido después de exposición antigénica específica (Korniluk et al, 2014). El CD27 ha sido ampliamente utilizado para distinguir los LB entre vírgenes y de memoria tanto en trabajos experimentales como en el contexto clínico (Vélez et al, 2015). Sin embargo, Fecteau, Roy, y Néron, (2009) observaron que la expresión de CD27 disminuye en los primeros días luego de la estimulación con CD40L+IL-4 y a partir del día noveno se restaura, sugiriendo así que se modula luego de la activación de los LB. También se ha descrito una población menor de LB de memoria IgG positivas sin la expresión de CD27 (10-20\%) (Tangye, y Good, 2007). Estos estudios demuestran que el uso de CD27 como único marcador para diferenciar los LB vírgenes y de memoria posee limitaciones. 
A.

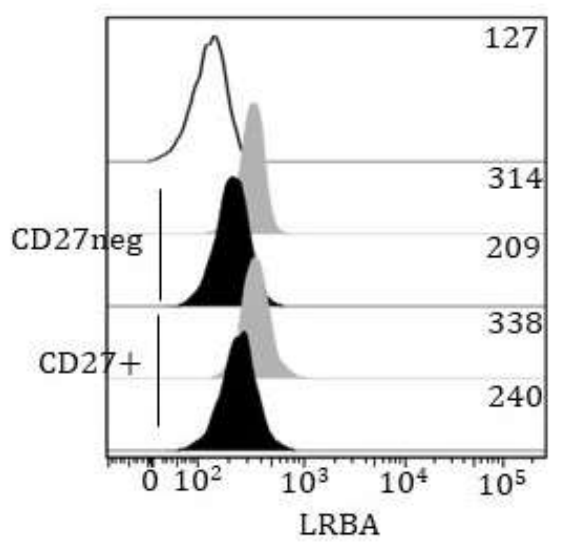

B.

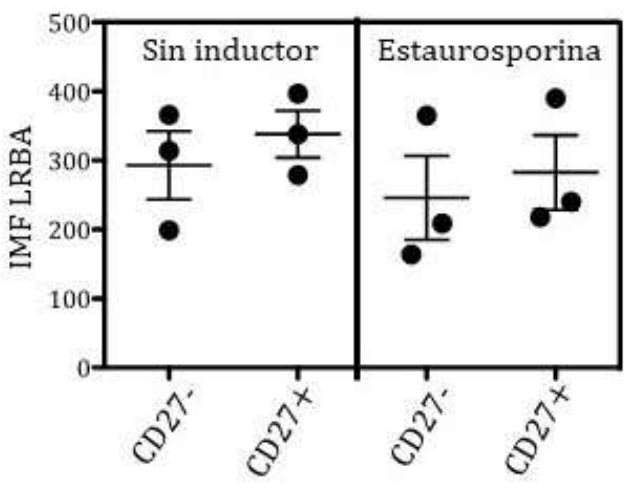

C.

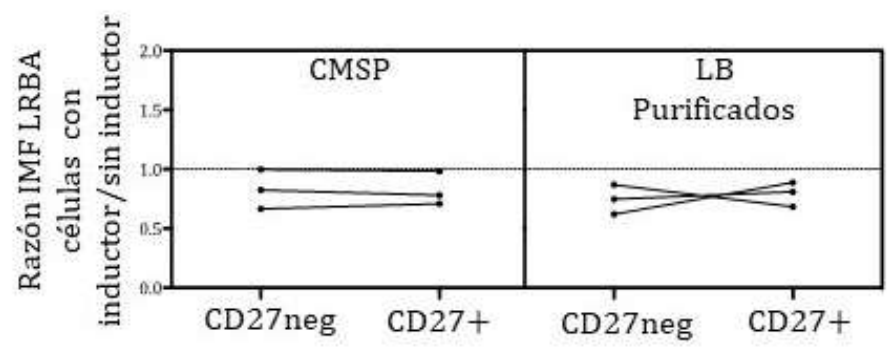

D.

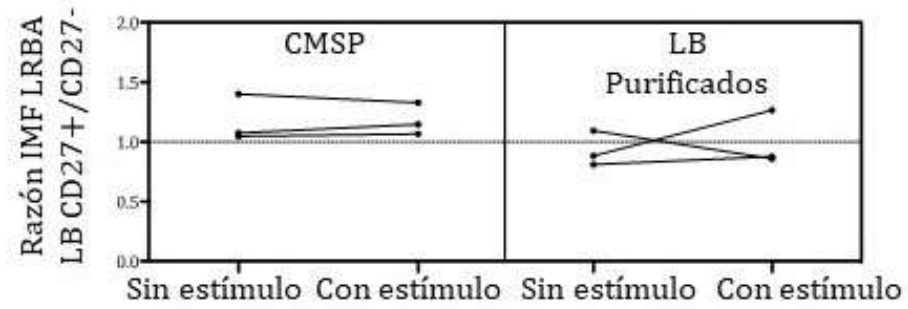

Figura 5. Expresión de LRBA linfocitos B vírgenes y de memoria obtenidas a partir de células mononucleares de sangre periférica expuestos a estaurosporina. A. Ejemplo representativo de la expresión de LRBA en linfocitos B vírgenes y de memoria obtenidos a partir de CMSP incubadas por $6 \mathrm{~h}$ en ausencia o presencia de estaurosporina. El histograma sólo delineado corresponde a la tinción únicamente con el anticuerpo secundario, los histogramas grises representan los resultados en linfocitos B no expuestos a estaurosporina y los histogramas negros en aquellos expuestos a estaurosporina B. Intensidad media de fluorescencia de los resultados obtenidos en los tres experimentos realizados. C. Razón de la intensidad media de fluorescencia de LRBA en linfocitos B ya sea vírgenes (CD27neg) como de memoria (CD27+) expuestos al inductor de apoptosis/aquellos sin el inductor por $6 \mathrm{~h}$, ya sea en linfocitos $\mathrm{B}$ a partir de células mononucleares de sangre periférica o purificados por separación electromagnética. D. Razón de la intensidad media de fluorescencia de LRBA en linfocitos B de memoria/linfocitos $B$ vírgenes en ausencia (sin inductor) o presencia (con inductor) de la estaurosporina por $6 \mathrm{~h}$, ya sea en linfocitos $\mathrm{B}$ a partir de células mononucleares de sangre periférica o purificados por separación electromagnética. Los resultados de cada experimento se encuentran unidos por una línea. La línea punteada indica igual expresión de LRBA en ambas subpoblaciones de linfocitos $B$. 
La mejor estrategia para caracterizar la memoria inmunológica en los LB de SP es la detección de hipermutación somática en la región variable de los genes de las inmunoglobulinas; sin embargo, esta metodología, además de ser dispendiosa y costosa, no permite la separación de las subpoblaciones para su posterior manipulación y análisis. Otra opción más viable para la discriminación de LB vírgenes y de memoria sería el uso adicional de marcadores como IgG, IgD e IgM junto con CD27 como en el estudio realizado por Pozo-Beltrán et al (2019). Sin embargo, la separación en subgrupos celulares de LB reduciría aún más el número de células disponibles para posteriores análisis por citometría.

López et al (2012) demostraron que la proliferación de los LB en pacientes deficientes de LRBA se afecta en respuesta a la estimulación mitogénica lo que sugiere un papel de esta proteína en este proceso. En el presente trabajo utilizamos el tri-CD40L e IL-21 para estimular los LB, debido a que, bajo nuestras condiciones experimentales, ha mostrado resultados satisfactorios (Erazo-Borras et al, 2017). En el presente trabajo se encontró que la expresión intracelular de LRBA con este estímulo se modula positivamente. De igual manera, en un estudio realizado por Gámez-Díaz et al (2018) evidenciaron que las CMSP estimuladas con fitohemaglutinina (PHA) aumentaban la expresión de la proteína LRBA en especial los LB.

En relación con la expresión intracelular de LRBA bajo condiciones de apoptosis, en nuestro estudio observamos que cuando los LB vírgenes y de memoria eran expuestos a estaurosporina, la expresión de LRBA se modulaba negativamente. Adicionalmente, en este estudio también observamos que la expresión de LRBA es mayor en los LB de memoria cuando estos se exponen a estaurosporina. Esto sugiere que se requiere de la participación y "gasto" de esta proteína en la supervivencia celular, como lo observaron Wang et al (2004), en un estudio realizado con células tumorales expuestas a estaurosporina y silenciando LRBA, en el cual se demostró que la ausencia de LRBA aumentaba la apoptosis en estas células. Pudiera ser también que si LRBA está implicada en la regulación de la apoptosis su expresión se vea reducida en estas condiciones por citometría de flujo posiblemente por la agregación y translocación de esta proteína al sistema de endomembranas, como lo observó previamente Wang, Howson, Haller, y Kerr (2001), al estimular líneas celulares de macrófagos con LPS y demostrar que había un cambio en el patrón de tinción vesicular después de la estimulación. Consideramos que se requiere de estudios complementarios que evalúen de manera cinética estas subpoblaciones en cultivo para aproximarnos aún más al papel de LRBA en la apoptosis celular de los LB y correlacionando la citometría de flujo, por ejemplo, con técnicas de microscopía de confocal o PCR en tiempo real durante la exposición a diferentes inductores de apoptosis.

Finalmente, nuestro hallazgo más importante fue la mayor expresión de LRBA in vitro en los LB de memoria de controles sanos bajo cualquiera de las condiciones utilizadas. Esta mayor expresión de LR$\mathrm{BA}$ en los LB de memoria podría explicar por qué los LB de los pacientes con deficiencia en esta proteína presentan defectos en la producción de inmunoglobulinas, en la diferenciación a plasmablastos, en la proliferación y en la supervivencia celular, funciones que tienen mayor relevancia en los LB de memoria. Lo et al (2015) identificaron que la deficiencia de LRBA causa una pérdida secundaria de CTLA-4 (Cytotoxic T-Lymphocyte Associated Protein 4) en los linfocitos $\mathrm{T}$ humanos activados, al afectar el tráfico intracelular de esta proteína, causando así una alteración en la homeostasis inmune de los linfocitos $\mathrm{T}$, lo que favorece el fenotipo de autoinmunidad en los pacientes deficientes de LRBA. Otro estudio previo demostró que mutantes dominantes negativas para LRBA disminuyen la expresión y la fosforilación de EGFR (Epidermal growth factor receptor) (Wang et al, 2004). La expresión del EGFR en la superficie celular conduce al reclutamiento de proteínas que activan las vías de señalización necesarias para la proliferación, diferenciación, crecimiento celular, migración e inhibición de la apoptosis de varios tipos de células, entre ellas los LB (Sigismund et al, 2008; Sorkin y Goh, 2008). Estos hallazgos sugieren que LRBA podría estar implicada en el tráfico vesicular o en la expresión extracelular de otras moléculas y/o receptores en los LB de memoria, dependientes del tráfico intracelular, esenciales para la proliferación, maduración y/o supervivencia de esta subpoblación 
celular.

En resumen, nuestros hallazgos apoyan la importancia de la proteína LRBA en la maduración y función principalmente de los LB de memoria activados. Estos hallazgos deben ser confirmados por medio de otras aproximaciones experimentales, por ejemplo, por medio de PCR en tiempo real o microscopia de fluorescencia. También es necesario evaluar cinéticamente la expresión de LRBA en estas subpoblaciones celulares de LB in vitro para dilucidar si los efectos de esta proteína se producen de manera temprana o tardía después de la activación. Finalmente, el silenciamiento de esta proteína en LB de memoria permitiría evaluar cuál de las funciones de estas células (proliferación, presentación antigénica, producción de anticuerpos, diferenciación) se ve mayormente afectada, por ejemplo, utilizando citometría de masas.

\section{AGRADECIMIENTOS}

Agradecemos al grupo de Inmunodeficiencias Primarias de la Universidad de Antioquia por permitir llevar a cabo este estudio. También agradecemos al Doctor Mauricio Rojas López por su ayuda y opinión experta en los ensayos basados en citometría de flujo. Este trabajo fue financiado por el Departamento Administrativo de Ciencia, Tecnología e Innovación, COLCIENCIAS (Proyecto 1115-569-34430).

\section{CONFLICTO DE INTERESES}

Los autores declaran no tener conflictos de intereses.

\section{REFERENCIAS}

Alkhair, O., Abolhassani, H., Rezaei, N., Fang, M., Kasper, V., Chavoshzadeh, Z., Mohammadzadeh, I., Mariam, A., Massaad, M., Chou, J., Aghamohammadi, A., y Geha, R. (2016). Spectrum of Phenotypes Associated with Mutations in LRBA. Journal Clinical Immunology, 36, 33. doi:10.1007/s10875-015-0224-7

Antonsson, A., y Persson, J.L. (2009). Induction of Apoptosis by Staurosporine Involves the Inhibition of Expression of the Major Cell Cycle Proteins at the G2/M Checkpoint Accompanied by Alterations in Erk and Akt Kinase Activities. Anticancer Research, 29, 2893-2898. http://ar.iiarjournals.org/content/29/8/2893.full
Antunes, R.F., Kaski, J.C., y Dumitriu, I.E. (2012). The Role of Costimulatory Receptors of the Tumour Necrosis Factor Receptor Family in Atherosclerosis. Journal of Biomedicine and Biotechnology, 464532. doi:10.1155/2012/464532

Brenner, D., Golks, A., Becker, M., Müller, W., Frey, C.R., Novak, R., Melamed, D., Kiefer, F., Krammer, P.H., y Arnold, R. (2007). Caspase-cleaved HPK1 induces CD95Lindependent activation-induced cell death in $\mathrm{T}$ and $\mathrm{B}$ lymphocytes. Blood, 110, 3968-3977. doi:10.1182/blood2007-01-071167

Cullinane, A.R., Schäffer, A.A., y Huizing, M. (2013). The BEACH is hot: a LYST of emerging roles for BEACHdomain containing proteins in human disease. Traffic, 14, 749-66. doi:10.1111/tra.12069

Erazo-Borras, L.V., Alvarez-Alvarez, J.A., Perez-Romero, C.A., Orrego-Arango, J.C., Franco-Restrepo, J.L., y Trujillo-Vargas, C.M. (2017). Skewed Invariant Natural Killer T (iNKT) Cells, Impaired iNKT:B Cell Help and Decreased SAP Expression in Blood Lymphocytes from Patients with Common Variable Immunodeficiency. Scandinavian Journal of Immunology, 86(3), 171-178. doi:10.1111/sji.12576

Espinosa, F.J., Condino, A., Franco, J.L., y Sorensen, R.U. (2016). En acción: para mejorar el acceso a la atención óptima para todos los pacientes con inmunodeficiencias primarias. Revista Alergia México, 63, 109-112. doi:10.18233/APM37No2pp64-68

Fecteau, J.F., Roy, A., y Néron, S. (2009). Peripheral blood $\mathrm{CD} 27+\mathrm{IgG}+\mathrm{B}$ cells rapidly proliferate and differentiate into immunoglobulin-secreting cells after exposure to low CD154 interaction. Immunology, 128(1 Pt 2), e353-e365. doi:10.1111/j.1365-2567.2008.02976.x

Gámez-Díaz, L., August, D., Stepensky, P., Revel-Vilk, S., Seidel, M.G., Noriko, M., Morio, T., Worth, A.J.J., Blessing, J., Van de Veerdonk, F., Feuchtinger, T., Kanariou, M., Schmitt-Graeff, A., Jung, S., Seneviratne, S., Burns, S., Belohradsky, B.H., Rezaei, N., Bakhtiar, S., Speckmann, C., Jordan, M., y Grimbacher, B. (2016). The extended phenotype of LPS-responsive beige-like anchor protein (LRBA) deficiency. The Journal of Allergy and Clinical Inmunology, 137, 223-230. doi:10.1016/j.jaci.2015.09.025

Gámez-Díaz, L., Sigmund, E.C., Reiser, V., Vach, W., Jung, S., y Grimbacher, B. (2018). Rapid Flow Cytometry-Based Test for the Diagnosis of Lipopolysaccharide Responsive Beige-Like Anchor (LRBA) Deficiency. Frontiers in Immunology, 9, 720. doi:10.3389/fimmu.2018.00720

Good, K.L., Bryant, V.L., y Tangye, S.G. (2006). Kinetics of Human B Cell Behavior and Amplification of Proliferative Responses following Stimulation with IL-21. Journal of Immunology, 177, 5236-5247. doi:10.4049/jimmunol.177.8.5236

Klein, U., Rajewsky, K., y Küppers, R. (1998). Human immunoglobulin $(\mathrm{Ig}) \mathrm{M}+\mathrm{IgD}+$ peripheral blood $\mathrm{B}$ cells expressing the CD27 cell surface antigen carry somatically mutated variable region genes: CD27 as a general marker for somatically mutated (memory) B cells. The Journal of Experimental Medicine, 188, 1679-1689. doi:10.1084/jem.188.9.1679

Korniluk, A., Kemona, H., y Dymicka-Piekarska, V. (2014). 
Multifunctional CD40L: pro- and anti-neoplastic activity. Tumor Biology, 35, 9447-9457. doi:10.1007/s13277-014$2407-\mathrm{x}$

Lo, B., Zhang, K., Lu, W., Zheng, L., Zhang, Q., Kanellopoulou, C., Zhang, Y., Liu, Z., Fritz, J.M., Marsh, R., Husami, A., Kissell, D., Nortman, S., Chaturvedi, V., Haines, H., Young, L.R., Mo, J., Filipovich, A.H., Bleesing, J.J., Mustillo, P., Stephens, M., Rueda, C.M., Chougnet, C.A., Hoebe, K., McElwee, J., Hughes, J.D., KarakocAydiner, E., Matthews, H.F., Price, S., Su, H.C., Koneti Rao, V., Lenardo, M.J., y Jordan, M.B. (2015). Patients with LRBA deficiency show CTLA4 loss and immune dysregulation responsive to abatacept therapy. Science, 349, 436-440. doi:10.1126/science.aaa1663

Lopez-Herrera, G., Tampella, G., Pan-Hammarström, Q., Herholz, P., Trujillo-Vargas, C.M., Phadwal, K., Simon, A.K., Moutschen, M., Etzioni, A., Mory, A., Srugo, I., Melamed, D., Hultenby, K., Liu, C., Baronio, M., Vitali, M., Philippet, P., Dideberg, V., Aghamohammadi, A., Rezaei, N., Enright, V., Du, L., Salzer, U., Eibel, H., Pfeifer, D., Veelken, H., Stauss, H., Lougaris, V., Plebani, A., Gertz, E.M., Schäffer, A.A., Hammarström, L., y Grimbacher, B. (2012). Deleterious Mutations in LRBA Are Associated with a Syndrome of Immune Deficiency and Autoimmunity. (2012). American Journal of Human Genetics, 90, 986-1001. doi:10.1016/j.ajhg.2012.04.015

Marasco, E., Farroni, C., Cascioli, S., Marcellini, V., Scarsella, M., Giorda, E., Piano Mortari, E., Leonardi, L., Scarselli, A., Valentini, D., Cancrini, C., Duse, M., Grimsholm, O., y Carsetti, R. (2017). B-cell activation with CD40L or CpG measures the function of B-cell subsets and identifies specific defects in immunodeficient patient. European Journal of Immunology, 47, 131-143. doi:10.1002/eji.201646574

Morbach H., Eichhorn, E.M., Liese, J.G., y Girschick, H.J. (2010). Reference values for B cell subpopulations from infancy to adulthood. Clinical and Experimental Immunology, 162, 271-279. doi:10.1111/j.13652249.2010.04206.x

Pozo-Beltrán, C.F., Suárez-Gutiérrez, M.A., YamazakiNakashimada, M.A., Medina-Vera, I., Saracho-Weber. F., Macías-Robles, A.P., Guzmán-Martínez, M.N., Navarrete-Rodríguez, E.M., Del Rio-Navarro, B.E., Espinosa-Padilla, S.E., y Blancas-Galicia, L. (2019). B subset cells in patients with chronic granulomatous disease in a Mexican population. Allergologia et Immunopathologia, 47, 372-377. doi:10.1016/j.aller.2019.03.005

Revel-Vilk, S., Fischer, U., Keller, B., Nabhani, S., GámezDíaz, L., Rensing-Ehl, A., Gombert, M., Hönscheid, A., Saleh, H., Shaag, A., Borkhardt, A., Grimbacher, B., Warnatz, K., Elpeleg, O., y Stepensky, P. (2015). Autoimmune lymphoproliferative syndrome-like disease in patients with LRBA mutation. Clinical Immunology, 159, 84-92. doi:10.1016/j.clim.2015.04.007

Sigismund, S., Argenzio, E., Tosoni, D., Cavallaro, E., Polo S., y Di Fiore, P.P. (2008). Clathrin-Mediated Internalization Is Essential for Sustained EGFR Signaling but Dispensable for Degradation. Developmental Cell, 15, 209-19. doi:10.1016/j.devcel.2008.06.012

Sorkin, A., y Goh, L.K. 2008. Endocytosis and intracellular trafficking of ErbBs. Experimental Cell Research, 314, 3093-3106. doi:10.1016/j.yexcr.2008.07.029

Tangye, S.G., y Good, K.L. (2007). Human $\operatorname{IgM}^{+} \mathrm{CD}_{2} 7^{+}$B Cells: Memory B Cells or "Memory" B Cells? Journal of Immunology, 179, 13-19. doi:10.4049/jimmunol.179.1.13

Vélez, A.C., Castaño, D.M., Gómez, R.D., Orrego, J.C., Moncada, M., y Franco, J.L. (2015). Inmunodeficiencia común variable: caracterización clínica e inmunológica de pacientes e identificación de subgrupos homogéneos con base en la tipificación de subpoblaciones de linfocitos B. Biomédica, 35, 101-16. doi:10.7705/biomedica.v35i1.2401

Wang, J.W., Howson, J., Haller, E., y Kerr, W.G. (2001). Identification of a Novel Lipopolysaccharide-Inducible Gene with Key Features of Both a Kinase Anchor Proteins and chs1/beige Proteins. Journal of Immunology, 166, 45864595. doi:10.4049/jimmunol.166.7.4586

Wang, J-W., Gamsby, J.J., Highfill, S.L., Mora, L.B., Bloom, G.C, Yeatman, T.J, Pan, T., Ramne, A.L., Chodosh, L.A., Douglas Cress, W., Chen, J., y Kerr W.G. (2004). Deregulated expression of LRBA facilitates cancer cell growth. Oncogene, 23, 4089-4097. doi:10.1038/sj.onc. 1207567

Wang, J-W., y Lockey, R.F. (2014). Lipopolysaccharideresponsive beige-like anchor (LRBA), a novel regulator of human immune disorders. Austin Journal of Clinical Immunology, 1, 1004. https://austinpublishinggroup.com/ clinical-immunology/fulltext/ajci-v1-id1004.php 